\title{
Isothiocyanato-Tolanes Based High Birefringence and Fast Response Time Mixtures for Photonic Applications
}

\author{
Sebastian Gauza \\ Chien-Hui Wen \\ Yang Zhao \\ Shin-Tson Wu \\ College of Optics and Photonics, University of Central Florida, \\ Orlando, Florida, USA
}

\section{Anna Ziółek \\ Roman Dabrowski}

Institute of Chemistry, Military University of Technology, Warsaw, Poland

We have developed some high birefringence $(\Delta n \sim 0.3$ at $\lambda=633 \mathrm{~nm})$ and relatively low viscosity nematic mixtures using isothiocyanato tolanes. These mixtures exhibit a high figure-of-merit ( $\left.F_{0} M\right)$ which is desirable for reducing the device response time. Using such a high FoM liquid crystal mixture in a 2- $\mu$ m homogeneous cell, we demonstrate a $1 \pi$ phase modulator at $\lambda=633 \mathrm{~nm}$ with submillisecond response time.

Keywords: high birefringence; nematic liquid crystals; photonic applications; response time

\section{INTRODUCTION}

Almost all the liquid crystal (LC) devices, such as display panels, spatial light modulators, and optical phased arrays for laser beam steering require a fast response time [1]. In order to achieve fast response time, low rotational viscosity LC mixtures are preferred

This work is supported by DARPA Bio-Optics Synthetic Systems program under Contract No. W911NF04C0048, and NATO Programme Security Through Science, Collaborative Linkage Grant No. CBP.EAP.CLG 981323.

Address correspondence to Sebastian Gauza, College of Optics and Photonics, University of Central Florida, Orlando, Florida 32816, USA. E-mail: swu@mail.ucf.edu 
[2-4]. Another straightforward approach is to use a thin cell gap filled with a high birefringence $(\Delta n)$ and relatively low viscosity LC mixture. The most effective way to increase birefringence is to elongate the $\pi$-electron conjugation length of the LC compounds.

Lately, several new high $\Delta n$ single LC compounds and nematic mixtures with relatively low viscosity, high resistivity, and good photo and thermal stabilities have been developed [5-7]. Fluoro (F), cyano $(\mathrm{CN})$, and isothiocyanato (NCS) are the three commonly employed polar groups. Among them, fluoro group possess a modest dipole moment, high resistivity, and low viscosity. However, its strong negativity compresses the electron clouds and, subsequently, lowers the compound's birefringence. As a result, the fluorinated compounds are more suitable for visible display applications where the required birefringence is around 0.1.

For infrared applications, a higher $\Delta n$ compound is needed in order to compensate for the longer wavelength. To obtain a higher birefringence, two approaches can be taken to enhance the electron conjugation length: 1) by elongating the core structure, e.g., tolane and terphenyl, and 2) by attaching an electron acceptor polar group, such as $\mathrm{CN}$ and NCS. The CN group has a larger dipole moment than NCS because of its linear structure. However, due to the very strong polarization of the carbon-nitrogen triple bond the Huckel charges of carbon and nitrogen are high and well localized. Accordingly, dimers are formed by the strong intermolecular interactions between the nitrile group and phenyl ring. Thus, a relatively high viscosity is observed in the cyanobased LC mixtures. On the other hand, the Huckel charges of nitrogen, carbon, and sulfur are smaller in the NCS group. The predicted intermolecular interactions by the NCS group in the isothiocyanato-benzene systems are smaller than those in the nitrile based systems. The dimers are not formed and, therefore, the viscosity of such molecular systems is lower than that of nitrile based ones [8]. Due to the longer $\pi$-electron conjugation, the NCS-based LC compounds exhibit a higher birefringence than the corresponding $\mathrm{CN}$ compounds.

In this paper, we investigate two groups of highly polar isothiocyanate compounds for display and photonics applications. Molecular structures, mesomorphic, and electro-optic properties of these single compounds and mixtures are reported. UV stability is addressed because it is a common concern for all the high birefringence LC molecular systems. For comparison between compounds and mixtures, we compare their Figure-of-Merit (FoM) which takes birefringence and visco-elastic coefficient into consideration. Potential applications of these mixtures for spatial light modulators, optical phased arrays, and high speed photonic devices are emphasized. 


\section{EXPERIMENT}

To measure the dielectric, elastic, optical, and electro-optic properties of LC compounds and mixtures, several instruments and experimental techniques are employed. For example, we used the capacitance method to measure the dielectric constants and Abbe refractometer to measure the individual refractive indices. Since the FoM only involves birefringence and visco-elastic coefficient, we could measure the phase retardation (for birefringence) and phase decay time (for visco-elastic coefficient) through a homogeneous alignment LC cell. In experiment, we prepared homogeneously aligned cells with cell gaps ranging from $\mathrm{d} \sim 4-8 \mu \mathrm{m}$ while a linearly polarized He-Ne laser $(\lambda=632.8 \mathrm{~nm})$ was used as the light source. A linear polarizer was placed at $45^{\circ}$ with respect to the LC cell rubbing direction and an analyzer was crossed. The light transmittance was measured by a photodiode detector (New Focus Model 2031) and recorded digitally by a LabVIEW data acquisition system (DAQ, PCI 6110 ). An ac voltage with $1 \mathrm{kHz}$ square waves was used to drive the LC cell whose inner surfaces were coated with ITO (indium-tin-oxide) electrodes. On top of the ITO, the substrates were overcoated with a thin polyimide alignment film. The buffing induced pretilt angle was about $2-3^{\circ}$. The cell was held in a Linkam LTS 350 Large Area Heating/Freezing Stage equipped with Linkam TMS94 Temperature Programmer. The phase retardation $(\delta)$ of the homogeneous cells was measured by the LabVIEW system. The LC birefringence $(\Delta n)$ at wavelength $\lambda$ and temperature T can be obtained by measuring the phase retardation $(\delta)$ of the homogeneous cell from the following Eq. [9]:

$$
\delta=2 \pi d \frac{\Delta n}{\lambda},
$$

By measuring the free relaxation (decay) time for a controlled phase change we can calculate the visco-elastic coefficient $\left(\gamma_{1} / K_{11}\right)$ according to the following equation:

$$
\tau_{o}=\frac{\gamma_{1} d^{2}}{K_{11} \pi^{2}}
$$

where $\gamma_{1}$ is rotational viscosity, $d$ is cell gap, and $K_{11}$ is the splay elastic constant. To compare the performance of LC mixtures, a Figureof-Merit (FoM) which takes phase retardation and visco-elastic coefficient into account is defined as [10]:

$$
\mathrm{FoM}=K_{11} \frac{(\Delta n)^{2}}{\gamma_{1}},
$$


where $K_{11}$ is the splay elastic constant, $\Delta n$ is the birefringence, and $\gamma_{1}$ is the rotational viscosity. All the three parameters in Eq. (3) are temperature dependent.

The dielectric anisotropy $(\Delta \varepsilon)$, threshold voltage $\left(\mathrm{V}_{\text {th }}\right)$, and elastic constants $\left(K_{11}, K_{33}\right)$ were measured by the LCAS II system (from LC Vision). All the measurements were conducted at room temperature of $23^{\circ} \mathrm{C}$ and the applied ac voltage frequency was $1 \mathrm{kHz}$ unless otherwise mentioned. All the thermal analyses were performed using a high sensitivity differential scanning calorimeter (DSC, TA Instrument Model Q-100). Phase transition temperatures were measured using small samples $(\sim 1.5 \mathrm{mg})$ at a $2^{\circ} \mathrm{C} / \mathrm{min}$ scanning rate. The observed LC phase transitions were confirmed by the polarizing optical microscopy (POM) method. For UV stability studies, the LC samples were illuminated with a Hamamatsu UV spot light source (Lightingcure L8868). The filter's transmittance range was $430-680 \mathrm{~nm}$. The LC cells with $\mathrm{SiO}_{\mathrm{x}}$ alignment layers were used for studying UV stability. The buffed polyimide cells are not recommended because the polyimide layer itself could be the instability factor [11].

\section{RESULTS AND DISCUSSION}

\section{A. Single Compounds}

Two groups of single compounds were investigated and their molecular structures are listed in Table 1. Most of the compounds, except $\mathbf{1}$ and $\mathbf{2}$, have the same rigid core which is NCS-tolane. Some compounds have an additional ring; either a saturated cyclohexyl ring or an unsaturated phenyl ring. The biphenyl-tolane structure has a longer $\pi$-electron conjugation than the cyclohexane tolane so that their birefringence is higher. However, cyclohexane helps to widen the nematic range which is important for mixture formulation. According to the molecular simulation results shown in Figure 1, the cyclohexyl ring does not lie in the same plane as the phenyl rings. The tolane unit which is formed by two phenyl rings linked by a triple bond, forms the main part of rigid core of the molecules and defines the principal molecular axis. Due to this bend structure, the cyclohexyl tolane has a larger visco-elastic coefficient $\gamma_{1} / K_{11}$ than its phenyl counterpart. The phenyl rings in the rigid core of the molecules are more or less in the same plane. Beside this unfavorable feature, the great benefit of using a cyclohexyl ring is its good mesomorphic property. Typically, such cyclohexane compounds exhibit only nematic phase above the melting point which happens in relatively low temperatures. The temperature range of the nematic could extend even to $200^{\circ} \mathrm{C}$ and 
TABLE 1 Molecular Structures Involved in BASE-1 and BASE-2 Mixtures

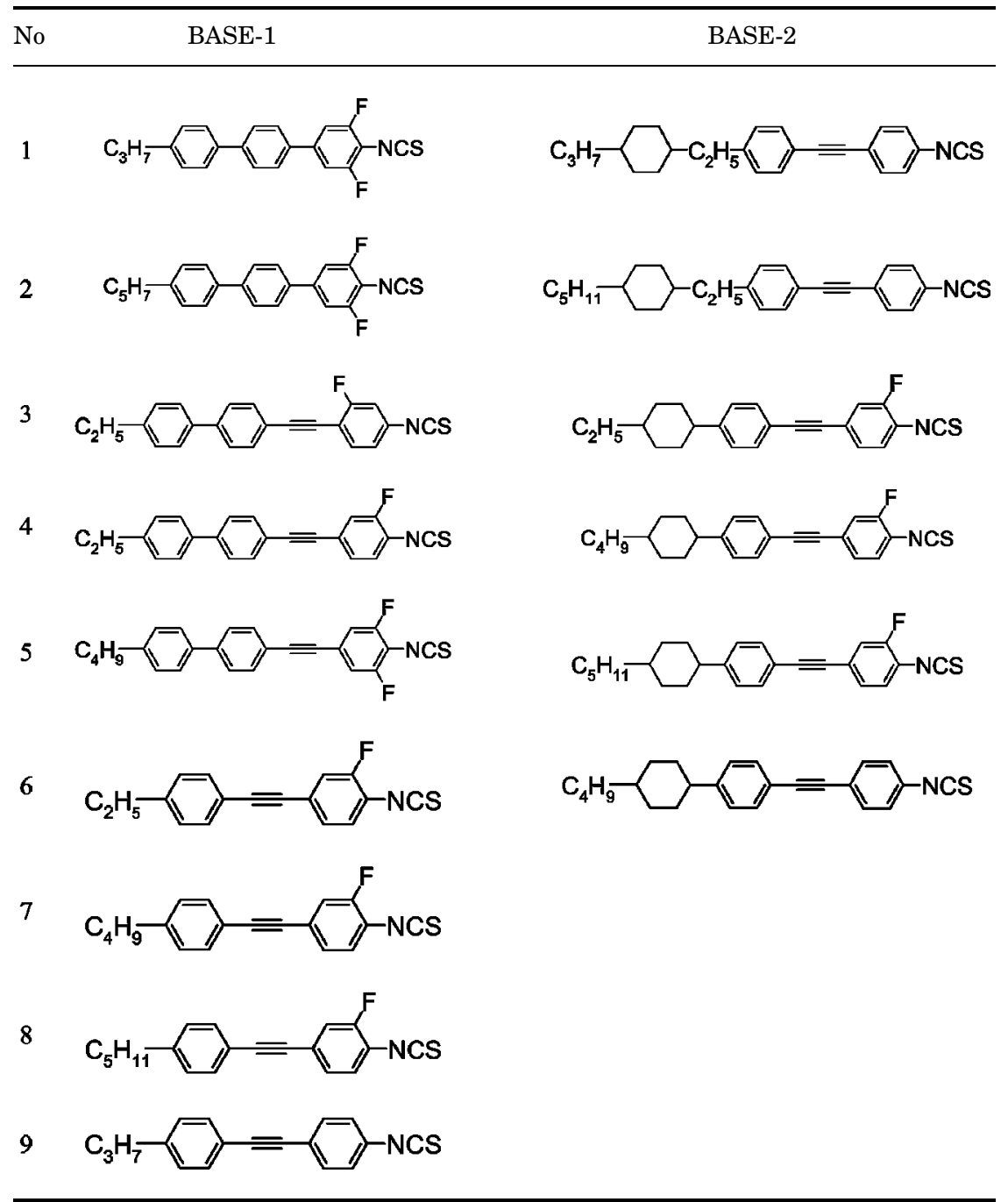

above, in some cases. Overall, these properties make the cyclohexyl compounds favorable for high birefringence eutectic mixtures. The compounds with a rigid core comprising of only phenyl rings show higher birefringence due to the longer $\pi$-electron conjugation. However, their mesomorphic properties are slightly worse than their cyclohexane analogs. Often, compounds based on the totally saturated rigid cores show highly ordered and thermally stable smectic E and B 


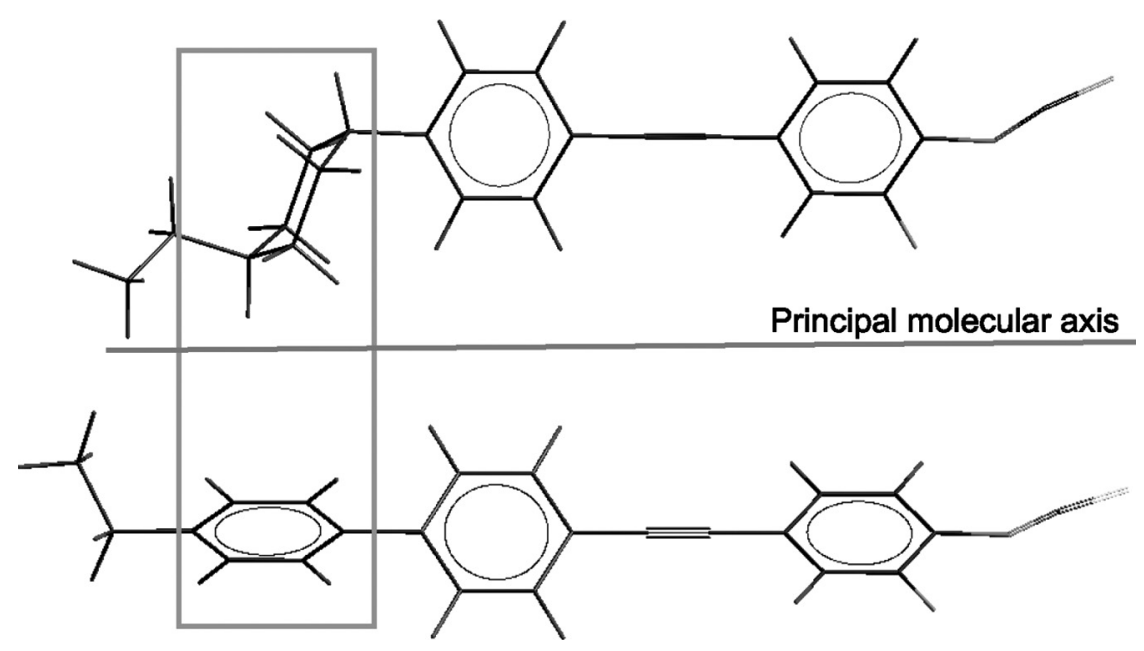

FIGURE 1 Simulated molecular structures of cyclohexyl and phenyl-based NCS tolanes. The Hyperchem v. 7 with MNDO method was used.

phases [6]. This unwanted phenomenon requires multiple lateral fluorinations in order to minimize the existence of the highly ordered smectic phases. The multiple lateral fluorinations lower slightly the optical anisotropy and increase the viscoelastic coefficient. The overall FoM of the totally aromatic compounds is higher than the ones based on cores with incorporated cyclohexyl ring [5].

The major challenges of high birefringence compounds are threefold: 1) higher melting temperature, 2) increased viscosity, and 3) weaker photostability. The high melting point results from the increased molecular linearity, rigidity, and polarity. The intermolecular interaction is stronger so that it requires a larger thermal energy to melt the molecules. High melting point is also unfavorable for mixture formulation. To overcome the high melting temperature problem, eutectic mixture needs to be formed. The increased viscosity originates from the bulkier molecular structure and larger moment of inertia. To reduce viscosity, we could consider operating the LC device at an elevated temperature. A rule of thumps is: as the temperature increases by $10^{\circ} \mathrm{C}$, the viscosity drops by $\sim 2 \mathrm{X}$. The reason that a high birefringence compound has a weaker photostability is because its UV absorption tail extends toward the visible spectral region. As a result, their long term photostability is a concern for display applications, especially in the blue wavelength. For infrared application, this concern is removed, as long as a UV filter is incorporated in front of the LC devices to filter out the harmful UV light. 


\section{B. Eutectic Mixtures}

To figure out the most efficient blend of good miscibility and mesomorphic performance with excellent FoM performance, we prepared two base mixtures, designated as BASE-1 and BASE-2. BASE-1 contains only totally aromatic terphenyls, phenyltolanes and tolanes while BASE-2 contains only cyclohexyl tolanes. The concentration dependent phase transition temperatures (called phase diagram) of the BASE-1 and -2 are shown in Figure 2. Two different smectic phases were observed under a polarizing optical microscope. The phase sequence of some of the compounds employed in BASE-1 shows a highly ordered smectic B phase while the compounds in BASE-2 shows tendency to form less ordered smectic A phase in the low temperature region. Two different smectic arrangements are destabilized in the direct neighborhood of the eutectic composition which leads to the pure nematic phase above the melting point [12]. The eutectic composition of BASE-1/BASE-2 appears at 55:45 $\mathrm{wt} \%$ ratios.

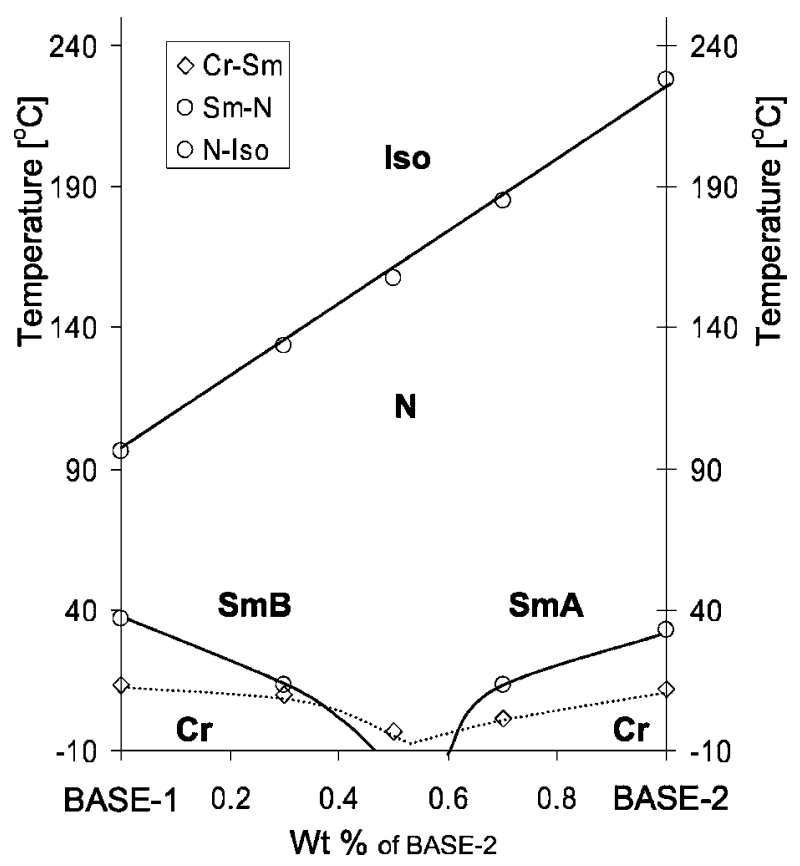

FIGURE 2 Phase transition temperature as a weight fraction of the BASE-1/BASE-2 system. 
The electro-optic performance of both BASE mixtures was measured at different temperatures. Temperature dependent birefringence is plotted in Figure 3(a). The solid lines represent fitting using the following formula:

$$
\Delta n=\Delta n_{o}\left(\frac{1-T}{T_{c}}\right)^{\beta}
$$

where $\Delta n_{o}$ is the birefringence at $\mathrm{T}=0 \mathrm{~K}, T$ is the operating temperature $(\mathrm{K}), T_{c}$ is the clearing temperature $(\mathrm{K})$, and $\beta$ is a material constant (typically $\sim 0.25$ ). The BASE- 1 mixture composed with only phenyl rings rigid core molecules has an initial $\Delta n_{o}$ higher than BASE-2 mixture which is based on the cyclohexyl tolanes. However, BASE-1 has a much lower clearing temperature due to the presence of two ring compounds so that its birefringence drops more quickly than BASE-2 as the temperature increases. The crossover temperature occurs at $45^{\circ} \mathrm{C}$.

Molecular structure of the compounds used for mixture formulation is a major factor affecting the rotational viscosity of the final mixture. As shown in Figure 3(b), the cyclohexyl tolane-based BASE-2 exhibits a higher visco-elastic coefficient than BASE-1. Consequently, the FoM of BASE-1, shown in Figure 3(c), is almost doubled as compared to that of BASE-2 in the $20-40^{\circ} \mathrm{C}$ temperature range. However, if the temperature is $\sim 20$ degrees below the clearing point of BASE- 1 , then BASE-2 has a higher FoM than BASE-1 because BASE-2's $\Delta n$ is still within the plateau region but the viscosity has been reduced dramatically.

Based on the phase diagram shown in Figure 2, we formulated two mixtures, designated as UCF-A and UCF-B, consisting of $70 / 30 \mathrm{wt} \%$ and $30 / 70 \mathrm{wt} \%$ of BASE- 1 and BASE-2 mixtures, respectively. We measured the temperature dependant $\Delta n, \gamma_{1} / \mathrm{K}_{11}$, and FoM for both mixtures and results are plotted in Figures 4(a)-4(c), respectively. This method provides a good flexibility for designing a mixture with optimal FoM at the desired operating temperature. The BASE-1 mixture which contains unsaturated phenyl tolanes offers a high FoM near the room temperature. On the other hand, the BASE-2 mixture which contains cyclohexane based structures is more desirable for operation at an elevated temperature. A high clearing temperature helps to boost FoM at an elevated temperature because of the lower viscosity.

By using the abovementioned principles we designed another mixture, UCF-C, intended for room temperature operation. The birefringence of UCF-C was adjusted to $\Delta n=0.30$ at $\mathrm{T} \sim 35^{\circ} \mathrm{C}$ and 

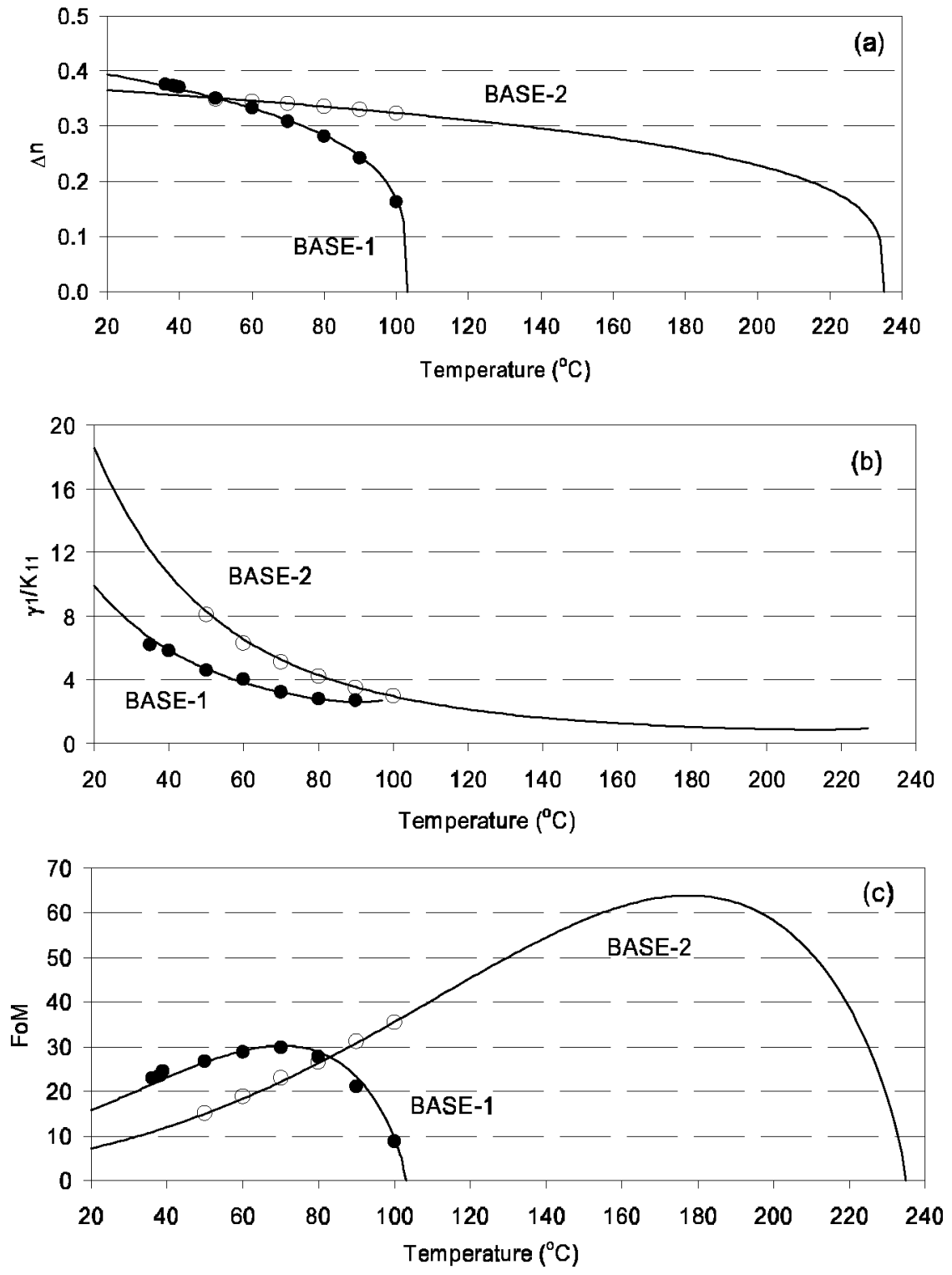

FIGURE 3 Temperature dependent (a) birefringence, (b) visco-elastic coefficient, and (c) figure-of-merit of BASE-1 and BASE-2 mixtures. 

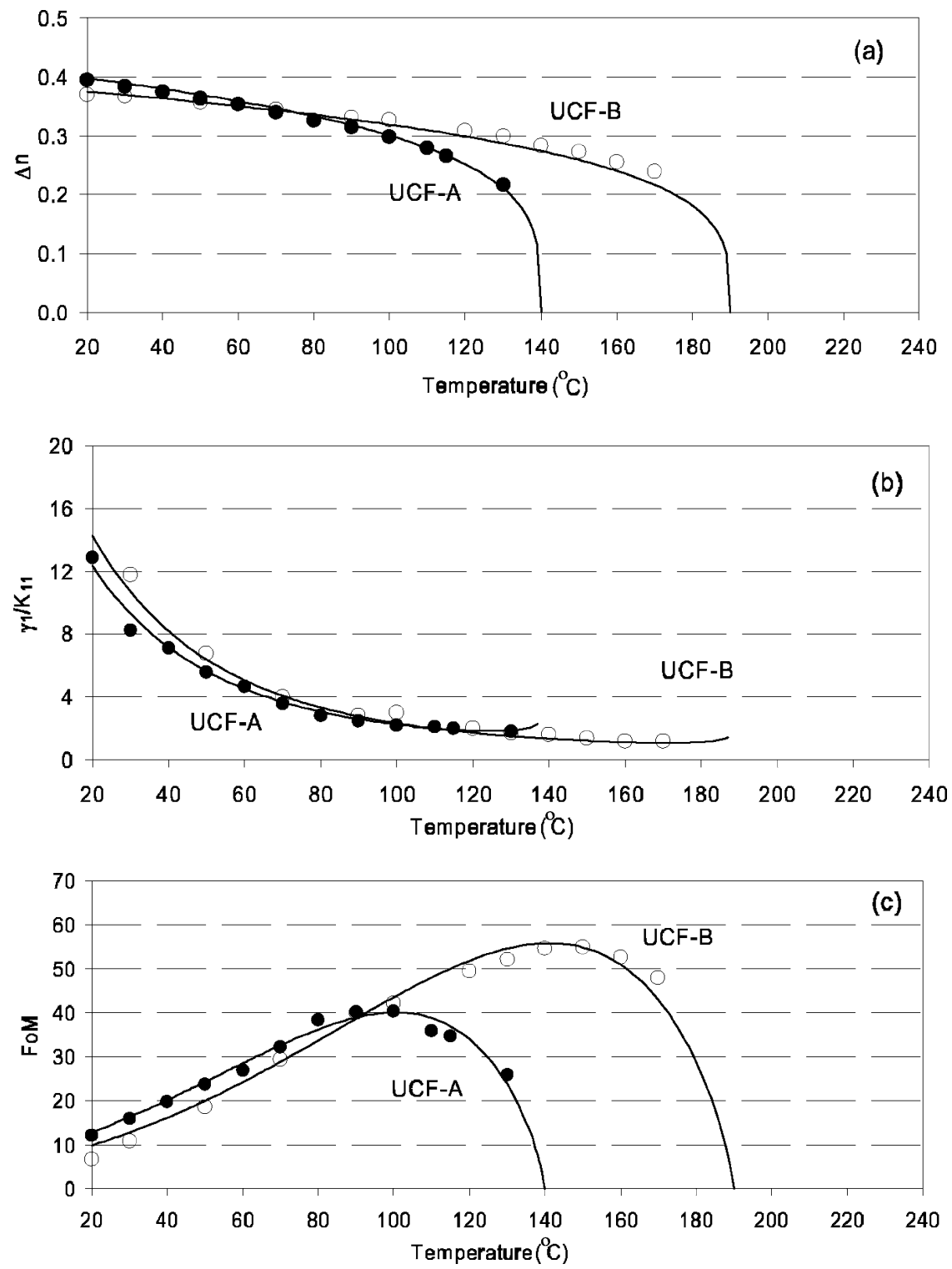

FIGURE 4 Temperature dependent (a) birefringence, (b) visco-elastic coefficient, and (c) figure-of-merit of UCF-A and UCF-B mixtures. 


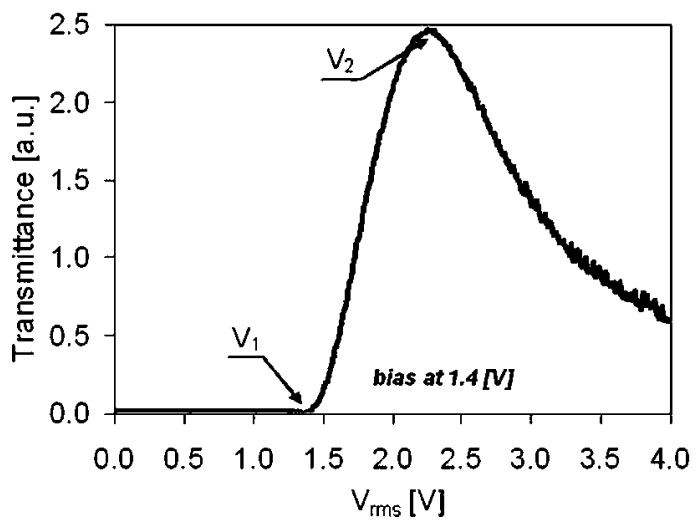

FIGURE 5 Voltage dependent transmittance of a homogenoeus cell sandwiched betwen crossed polarizers. $\lambda=633 \mathrm{~nm}$. $\mathrm{V}_{1}$ is the biased voltage and $\mathrm{V}_{2}$ is the on-state voltage. The LC mixture is UCF-C.

$\lambda=633 \mathrm{~nm}$. To take the advantage of high birefringence of our test mixture and realize the fast switching LC phase modulator, we injected the mixture into a $2 \mu \mathrm{m}$ thick homogeneous cell.

Figure 5 plots the voltage-dependent transmittance at $\lambda=633 \mathrm{~nm}$ of the UCF-C cell between crossed polarizers. For a homogeneous cell, the voltage-off state depends on the total phase retardation and may not be dark. In Figure 5, the voltage-off state is not perfectly dark. Thus, we applied a bias voltage at $\mathrm{V}_{1}=1.4 \mathrm{~V}_{\mathrm{rms}}$, which is slightly above the threshold voltage $\left(\sim 1.38 \mathrm{~V}_{\text {rms }}\right)$. The cell was switched

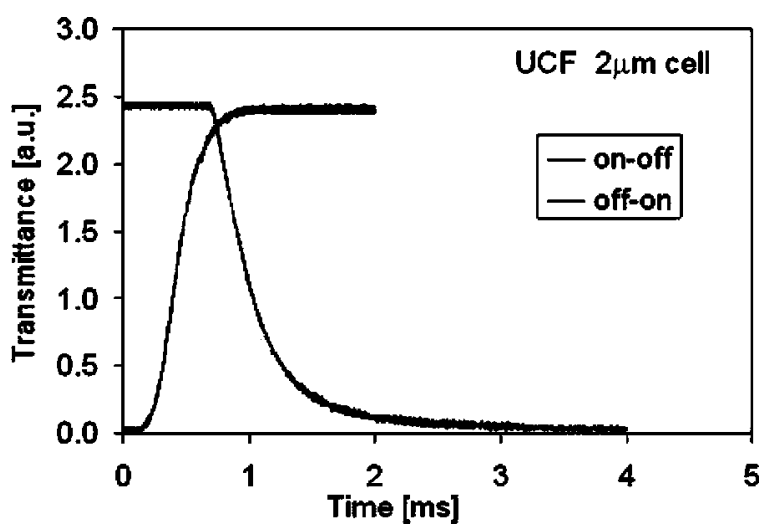

FIGURE 6 Switching time measured for on-off-on sequence of the optimized mixture filled into a $2 \mu \mathrm{m}$ homogeneous cell. Cell temperature was stabilized at $35^{\circ} \mathrm{C}$. 
between $\mathrm{V}_{1}$ and $\mathrm{V}_{2}\left(\sim 2.3 \mathrm{~V}_{\mathrm{rms}}\right)$. Although the way we measured the onoff and off-on response times is not commonly practiced, it shows that fast switching could be obtained if the cell gap was reduced through the use of a high birefringence mixture. The dark-to-bright $\left(V_{1} \rightarrow V_{2}\right)$ and bright-to-dark $\left(\mathrm{V}_{2} \rightarrow \mathrm{V}_{1}\right)$ switching times were measured to be $850 \mu \mathrm{s}$ and $420 \mu \mathrm{s}$, respectively, as shown in Figure 6.

\section{CONCLUSION}

We have measured and compared the $\Delta n, \gamma_{1} / K_{11}$ and FoM values for two different classes of highly conjugated isothiocyanates. Based on the direct FoM comparison of the investigated LC compounds, we conclude that the mixtures consisting of totally aromatic two and three rings compounds are more favorable for room temperature operation. The advantages are twofold: lower viscosity and higher birefringence. The shortcomings of such system are their relatively low clearing temperature and strong smectogenity. Thus for the mixtures intended for an elevated temperature operation, some compounds, e.g., cyclohexane tolanes, with a high clearing temperature should be incorporated in order to boost the clearing temperature. The mixtures we prepared here show a high birefringence and low viscosity and, therefore, their FoM is reasonably large. This would lead to a fast response time if such a LC is used in a thinner cell. The example demonstrated in our experiment shows submillisecond rise and decay times using a $2-\mu \mathrm{m}$ test mixture at $\mathrm{T}=35^{\circ} \mathrm{C}$. Potential applications of this kind of mixtures for various photonic devices are foreseeable.

\section{REFERENCES}

[1] Wu, S. T. \& Yang, D. K. (2001). Reflective Liquid Crystal Displays, Wiley: New York.

[2] Kibe, S., Hattori, N., Ushioda, M., Yamamoto, H., \& Matsui, S. (2003). J. SID, 11, 449.

[3] Geelhaar, T., Tarumi, K., \& Hirschmann, H. (1996). SID Symposium Digest, 27, 167.

[4] Goto, Y., Ogawa, T., Sawada, S., \& Sugimori, S. (1991). Mol. Cryst. Liq. Cryst., 209, 1.

[5] Gauza, S., Wang, H., Wen, C. H., Wu, S. T., Seed, A. J., \& Dabrowski, R. (2003). Jpn. J. Appl. Phys., Part 1, 42, 3463.

[6] Spadlo, A., Dabrowski, R., Filipowicz, M., Stolarz, Z., Przedmojski, J., Gauza, S., Fan, Y. H., \& Wu, S. T. (2003). Liq. Cryst., 30, 19.

[7] Gauza, S., Wen, C. H., Tan, B. J., \& Wu, S. T. (2004). Jpn. J. Appl. Phys., 43, 7176.

[8] Malecki, J. A. \& Nowak, J. (1999). J. Molecular Liquids, 81, 245.

[9] Wu, S. T., Efron, U., \& Hess, L. D. (1984). Appl. Opt., 23, 3911.

[10] Wu, S. T., Lackner, A. M., \& Efron, U. (1987). Appl. Opt., 26, 3441.

[11] Wen, C. H., Gauza, S., \& Wu, S. T. (2005). J. SID, 13, 805-811.

[12] Czupryński, K. (1990). Mol. Cryst. Liq. Cryst., 192, 47. 